Virtual Mentor. February 2004, Volume 6, Number 2.

doi: 10.1001/virtualmentor.2004.6.2.fred1-0402

From the Editor

\title{
Towards Defining Paternalism in Medicine
}

\section{The theme editor introduces an issue focusing on paternalism in medical decision making.}

It is 1804. A physician peers over the rims of his spectacles at his patient. She says, "Doctor, I don't want to die. Please tell me what to do." He replies, "Mrs. Smith, we will treat your illness with these leeches, which will cleanse your blood of the disease. That, combined with cold water dousing each night, will cure you."

In Southern California 2 hundred years later, a cancer patient says, "Doctor, I don't want to die. I've heard about a new experimental treatment being tested at Stanford, and I want to enroll in their study. If that doesn't work, then I want to get my nutritionist and a herbalist involved."

The history of medicine has witnessed a gradual erosion of the physician's time-honored role as all-knowing healer. Whether physicians were experts in their fields, self-taught folk healers, or complete quacks, the doctor's words, for generations, were accepted as correct, complete, final, and to be obeyed. Indeed, the language of the 1847 Code of Medical Ethics of the American Medical Association, titled "Obligations of Patients to Their Physicians", endorsed this paradigm.

The obedience of a patient to the prescriptions of his physician should be prompt and implicit. He should never permit his own crude opinions as to their fitness, to influence his attention to them. A failure in one particular may render an otherwise judicious treatment dangerous, and even fatal [1].

The patient was treated like a child; innocent, unschooled, and too simple to know how to take care of himself or herself. This wise father-simple child relationship led to an inherently paternalistic model of the physician-patient relationship.

But while science and technology have filled medical books with more and more treatment options and diseases are better understood, the instantaneous dissemination of news around the world has simultaneously rendered the public hyper-aware of the new capabilities of medicine. As a result, patients have shifted from approaching physicians with hope and faith to approaching them with high expectations - of precision, of speed, of a virtual superstore of treatment options.

Patients have taken the reins of health care with both hands. They come to doctor's offices armed with reams of printouts from health Web sites. They specifically request medicines or treatments advertised in popular magazines, on television, and on the Internet. In response to this type of informed (though sometimes misinformed) patient, many physicians have come to grant a greater level of autonomy or shared decision making to all the patients in their practices.

A turning point in the shift from physician paternalism to respect for patient autonomy was the requirement for the patient's informed consent to treatment. The concept of informed consent did not exist in writings on Egyptian, Greek, or Roman medicine. Indeed, the phrase "informed consent" was not used until the 1950s. The notion of "consent to treatment" was a consequence of the Nuremberg Trials that later became enshrined in the research and treatment codes of democratic nations.

Today, as any medical student or resident can tell you, most procedures in a hospital are preceded by explanations and 
discussions at the patient's bedside that make clear all the risks and benefits of the procedure. The consent conversation must be conducted by an $\mathrm{MD}$, and the patient must be able to understand what he or she is being asked to agree to.

Reflecting the importance of informed consent in modern health care, an opinion from the current AMA Code of Ethics, on "Fundamental Elements of the Patient-Physician Relationship" states: "The patient has the right to make decisions regarding the health care that is recommended by his or her physician. Accordingly, patients may accept or refuse any recommended medical treatment" [2].

Society's awareness of this change in approach to the patient has altered the connotation of the word 'paternalism' and, hence, popular perception of the physician who acts in a paternalistic manner. Paternalism in a physician is often described in a negative context, indicating inappropriately archaic behavior.

The dramatic changes in the role and perception of paternalism in medicine have complicated the medical field that residents and medical students are trying to navigate. Students at the University of California, Irvine, are taught the "Five E's" of effective patient-centered communication from day 1 of medical school (engage, empathize, educate, enlist, and extend). Concepts such as the five E's illustrate a shift in power to the patient and the requirement that the physician adapt to that patient. While medical training can theoretically teach such adaptability through example, ensuring that programs turn out nonpaternalistic physicians is something of a challenge. The older generation of physicians training younger residents and students comes from a time when paternalism was the norm in good medical practice. Physicians of today must acknowledge this changing tide while exerting enough authority to ensure that patients suffer no harm and receive the best medical care possible.

This issue of Virtual Mentor gives us perspectives from various points along the long and tortuous continuum from absolute patient autonomy to outright physician paternalism. We seek to illustrate the range of possibilities for shared decision making and how each situation should dictate the exact combination of patient and physician input.

We present 3 hypothetical clinical cases for comment, including the dilemma of a physician caught between her obligation to help her patient and her reservations about recurrent abortions, the case of a patient who tries to make a deal with his doctor to delay medical treatment for the sake of convenience, and, finally, a situation in which parents wish to convince their child's physician to accept their treatment decision.

The Policy Forum questions whether resorting to quasi-legal statements of patients' "rights" is the most effective means for securing patient autonomy. The Medicine and Society articles conjecture that unfettered patient autonomy is not necessarily a good thing, and that patients who desire full autonomy may be missing out on the education and perspective that are valuable components of a physician office visit. The complex case of a patient who is incompetent is explored in the Health Law section.

We hope that this discussion of physician and patient decision making will inform our readers and provoke discussion among practicing physicians, residents, and medical students. We welcome and encourage comments and questions.

The learning objectives for this issue are:

1. Understand what makes a medical decision or action paternalistic.

2 . Understand the range of possibilities for shared decision making by patient and physician.

3. Recognize situations that call for the physician to take a dominant role in decision making.

4. Understand the requirements for true autonomous decision making.

Sincerely,

Monya De 


\section{References}

1. American Medical Association. Article II, Obligation of patients to their physicians. Code of Medical Ethics. Chicago: AMA Press; 1847. Accessed February 2, 2004.

2. Opinion 10.01 Fundamental Elements of the Patient-Physician Relationship. Code of Medical Ethics 2008-2009 Edition. Chicago, IL: American Medical Association; 2008:341-347.

The viewpoints expressed on this site are those of the authors and do not necessarily reflect the views and policies of the AMA.

(C) 2004 American Medical Association. All Rights Reserved. 